\title{
Numerical study of the flow and passive scalar transport in an open-channel confluence with a flat and a degraded fixed bed
}

\author{
T. Jin, P.X. Ramos, L. Schindfessel and T. De Mulder \\ Hydraulics Laboratory, Dep. Civil Engineering, Ghent University, Ghent, Belgium
}

\begin{abstract}
Confluences are important junctions in fluvial and artificial networks of open-channels, as they regulate the mixing phenomena of substances transported by the merging flows, as well as the scour and deposition phenomena. This paper aims at contributing to the study of how the bed morphology, such as the presence of a scour hole and a depositional bar, influences the flow and mixing phenomena at a T-shaped open-channel confluence. By means of Large Eddy Simulations (LES) of flow and passive scalar transport, a comparative numerical study is carried out of two fixed bed cases, i.e. a flat one and a degraded one, which were investigated earlier experimentally in the lab and numerically with a RANS based solver by Tang et al. (2018). The spatial focus of the present paper is not exclusively devoted to the Confluence Hydrodynamics Zone, but also extends somewhat further downstream (i.e. up to 10 post-confluence channel widths $W_{d}$ downstream of the upstream confluence corner). For the investigated flow condition with a dominant incoming flow from the upstream main channel, the recirculation zone in the degraded bed case is found to be significantly shorter and less wide or even absent near the bed, yielding differences in the contraction of the main channel flow and in the secondary flow. Nevertheless, the non-uniformity of passive scalar mixing was found to be very similar up to $3.5 W_{d}$. Further downstream, the flat bed case shows a slightly higher mixing rate, which does not seem to be related to differences in secondary flow patterns, but rather to differences in turbulence levels.
\end{abstract}

\section{INTRODUCTION}

Confluences are key features of fluvial and artificial networks of open-channels, as they regulate the mixing phenomena of the incoming flows, that often transport different loads of dissolved or suspended substances (nutrients, pollutants, sediments, ...), as well as scour phenomena and deposition of sediments (Rice et al., 2008). Though these phenomena are often studied in the field (see e.g. Rhoads and Johnson, 2018), the confluence hydrodynamics zone (CHZ) is also studied by means of laboratory experiments and numerical modelling, in schematized geometries consisting of straight branches with rectangular cross-sections and sharp junction corners, see e.g. Best (1987), Weber et al. (2001), Bradbrook et al. (2001), Biron et al. (2004), Guillén-Ludeña et al. (2015), Yuan et al. (2018). The flow features, and the associated mixing phenomena, turn out to be influenced by geometrical parameters (such as confluence angle, discordance of channel widths, discordance in bed elevation), as well as hydraulic parameters (such as ratio of incoming momentum fluxes, ratio of width to flow depth, downstream Froude number). The present paper aims at contributing to the study of how the bed morphology, like e.g. the presence of a scour hole and a depositional bar, influences the flow and mixing phenomena at a T-shaped open-channel confluence (Figure 1). More specifically, a comparative numerical study will be carried out of two fixed bed cases, i.e. a flat one and a degraded one, which were investigated earlier experimentally and numerically by Tang et al. (2018). In contrast to their RANS based simulations, an eddy-resolving approach will be adopted in the present work. Both the flow and the mixing of a passive scalar will be investigated by means of Large Eddy Simulation (LES). Moreover, the spatial focus of this paper will not be mainly devoted to the CHZ, as in Tang et al. (2018), but will also extend somewhat further downstream, though not as far as needed for studying the length for complete mixing, as in Pouchoulin et al. (2018). 


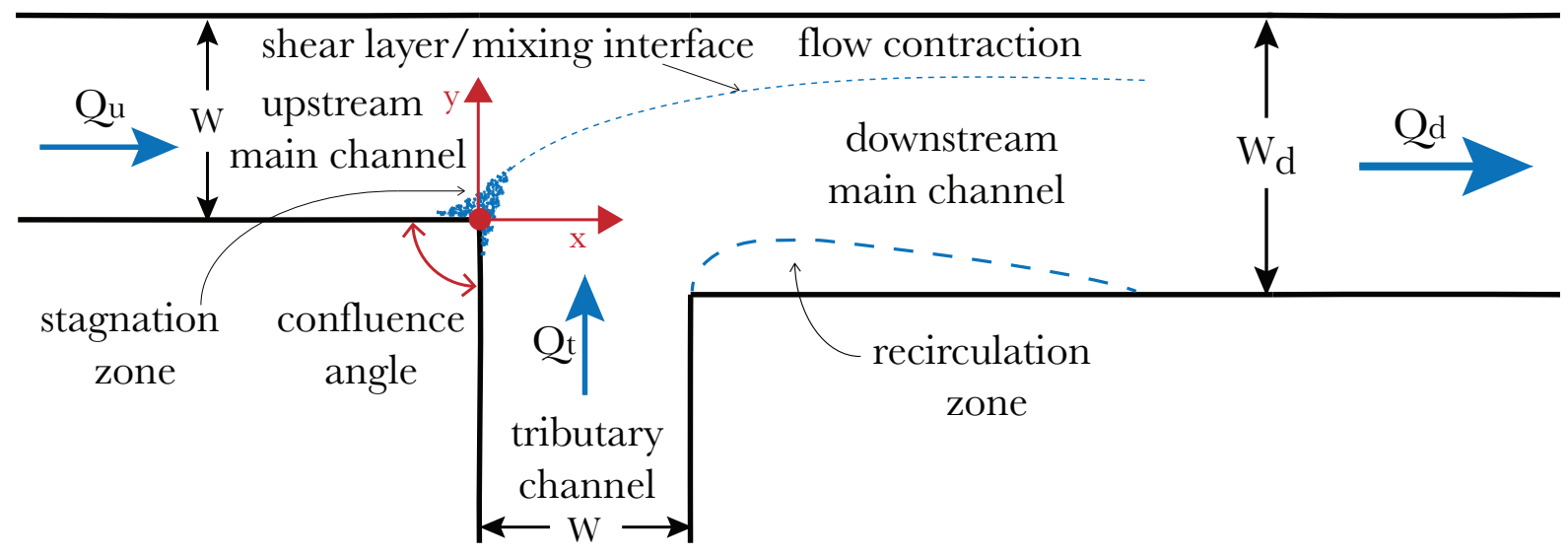

Figure 1. Schematic plan-view of a $90^{\circ}$ angled open-channel confluence flow.

\section{HYDRAULIC CONDITIONS}

Tang et al. (2018) carried out laboratory experiments in their right-angled confluence flume, consisting of channels with a rectangular cross-section. The downstream (i.e. post-confluence) channel width $W_{d}(=0.4 m)$ is larger than the width $W(=0.3 m)$ of the main upstream channel and the tributary channel, as shown schematically in Figure 1. The associated width discordance ratio $\omega\left(=W / W_{d}\right)$ is 0.75 . In the present work, only one flow condition of Tang et al. (2018) (their case 1) is considered, characterized by a downstream flow depth $h_{d}=0.163 \mathrm{~m}$ and the following flow rates in the upstream main channel, tributary channel and downstream main channel, respectively: $Q_{u}=9.0 \mathrm{~L} / \mathrm{s}, Q_{t}=6.0 \mathrm{~L} / \mathrm{s}$ and $Q_{d}=\left(Q_{u}+Q_{t}\right)=15.0 \mathrm{~L} / \mathrm{s}$. The corresponding cross-sectionally averaged downstream velocity $U_{d}\left(=Q_{d} /\left(h_{d} W_{d}\right)\right)$ is $0.23 \mathrm{~m} / \mathrm{s}$, the downstream Froude number $F r_{d}\left(=U_{d} /\left(g h_{d}\right)^{1 / 2}\right)$ is 0.18 , the discharge ratio $q\left(=Q_{u} / Q_{d}\right)$ is 0.6 , the ratio of incoming discharges $Q_{t} / Q_{u}$ is $2 / 3$ and the ratio of incoming momentum fluxes $M\left(=Q_{t}^{2} / Q_{u}^{2}\right)$ is $4 / 9$. In this work, both fixed bed experiments that were carried out by Tang et al. (2018) under this flow condition will be simulated: the flat bed case and the degraded bed case. The flat bed case consists of a horizontal bed of poorly sorted sand with $d_{50}=0.9 \mathrm{~mm}$ and $d_{90}=2.5 \mathrm{~mm}$. The degraded bed case consists of an equilibrium bed which developed from a layer of the same sandy material. The bathymetry of the degraded bed is characterized by a scour hole (with maximum depth $\approx 0.20 h_{d}$ ) and a depositional bar (with maximum height $\approx 0.25 h_{d}$ ), see Figure 2 .

\section{NUMERICAL METHODOLOGY}

\subsection{Large-Eddy Simulations within OpenFOAM}

The turbulent open-channel flow is modelled by means of Large Eddy Simulations (LES), adopting a standard Smagorinsky Subgrid-Scale (SGS) model with a constant $C_{s}$ of 0.158 (Rodi et al., 2013), making use of the OpenFOAM toolbox. The governing Navier-Stokes and continuity equations are discretized using the Finite Volume Method (FVM). The employed discretization schemes are second order accurate in time and space and the discretized equations are coupled and solved using the PISO algorithm. To simulate the mixing of a passive scalar in the flow, the LES approach will be adopted with a dynamic SGS model (Rodi et al., 2013). The governing transport equation is discretized by means of second order accurate schemes in time and space.

\subsection{Boundary conditions}

To treat the free surface, the rigid-lid approach will be adopted, implying that the free surface is replaced by a frictionless and impermeable upper boundary of the computational domain. More specifically, the curved rigid-lid approach described in Ramos et al. (2019a) is adopted in the present work. The curved shape of the upper domain boundary is defined by the location of a virtual free surface which would cause a hydrostatic pressure field identical to the time-averaged pressure field predicted on a flat rigid-lid in a preceding LES simulation. The wall boundary layers will not be fully resolved. Wall functions are 
adopted as in Schindfessel et al. (2015). The lateral walls of the flume are modelled as smooth walls, whereas the bed is modelled as a rough wall with a roughness height $k_{s}$. In the literature, different recommendations are available to express $k_{s}$ as a multiple of a typical grain size diameter of the sandy bed (e.g. Sleath, 1984). In the present work, a sensitivity analysis was carried out on $k_{s}$. A slightly better agreement with the available experimental velocity data for the degraded bed case in Tang et al. (2018) was found near the bed for $k_{s}=0.8 \mathrm{~mm}\left(\approx 1.25 d_{35}\right)$, in comparison to $k_{s}=2.5 \mathrm{~mm}\left(\approx d_{90}\right)$. Note that this $0.8 \mathrm{~mm}$ value for $k_{s}$ is relatively low as compared to the size of the large sand grains $\left(\sim d_{90}\right)$, which may be expected to armour the bed at the end of the development of the degraded bed. This lowering of the roughness height may be due to the fixation of the equilibrium bed sand grains, prior to the velocity measurements. Since the fixation is also adopted prior to the flat bed case measurements, the same roughness height $k_{s}=0.8 \mathrm{~mm}$ will also be adopted for the flat bed case simulation. Since a LES resolves a relatively big part of the turbulence on the mesh, and to approach the model to the experimental conditions at the inlets of the computational domain, the inlet velocity should also be turbulent and fully developed. In the present numerical set up, this is achieved by means of a so-called precursor simulation, which basically means that a periodic channel is simulated in the beginning of each upstream channel, and its turbulent velocity is used as an inlet condition. For the pressure variable, a zero value is imposed at the outlet and a zero gradient at the inlets $\left(x / W_{d}=-5\right.$ and $y / W_{d}=-5$; see Figure 1 for definition of coordinate system), the walls and the rigid-lid. For the subgrid-scale viscosity a zero gradient is imposed at all boundaries, except at the walls, where the aforementioned wall functions are adopted. As to the passive scalar transport LES simulations, the inlet boundary conditions for the concentration are an (arbitrary) uniform value of $100 \mathrm{mg} / \mathrm{L}$ for the tributary inlet and 0 for the upstream main channel inlet, while for the outlet a zero gradient condition is imposed. These conditions correspond to the $S 1 / S 2$ scenario investigated numerically by Tang et al. (2018) with a RANS based solver.

\subsection{Computational mesh}

A block-structured mesh used for the present numerical investigation has been defined (Table 1) after a mesh sensitivity analysis of the results. Grading of the cell size is adopted, yielding a higher resolution in the confluence zone and a smooth transition between the different blocks. Our mesh sensitivity analysis shows that a coarser resolution than adopted in this paper will miss the secondary currents, like it is reported for another open-channel confluence in Ramos et al. (2019a), and in open-channel flows in general, by Talebpour and Liu (2019). Therefore, special care was devoted to the mesh independence in terms of secondary flow results. The mesh for the flat bed case simulation is deformed for the subsequent degraded bed case simulation, based upon the following deformed mesh methodology. Along each vertical grid line (i.e. the $\mathrm{z}$ direction), the lowest grid point is shifted to coincide with the degraded bed bathymetry digitized from the experimental dataset of Tang et al. (2018), whereas the lowest-butone grid point is shifted while maintaining the same distance to the bed, in order to (approximately) apply the wall functions under circumstances comparable to the flat bed case. The other grid points in between the (curved) rigid-lid and the lowest-but-one grid point are gradually redistributed along the vertical gridline (Figure 2, left). The quality of the deformed mesh still meets the quality criteria (in terms of maximum aspect ratio, non-orthogonality and maximum skewness) checked by OpenFOAM.

Table 1. Number of mesh cells in both flat bed and degraded bed cases.

\begin{tabular}{lllllll}
\hline & \multicolumn{3}{c}{ Upstream channels } & \multicolumn{3}{c}{ Downstream main channel } \\
Spatial dimension & Longitudinal & Lateral & Vertical & Longitudinal & Lateral & Vertical \\
\hline Length scale & $5 W_{d}$ & $0.75 W_{d}$ & $\sim h_{d}$ & $10 W_{d}$ & $W_{d}$ & $\sim h_{d}$ \\
Number of cells & 600 & 80 & 30 & 850 & 100 & 30 \\
\hline
\end{tabular}

\subsection{Simulation time}

To obtain the LES results presented in this paper, the simulations have advanced 500 seconds, before the data collection started. This initialization time corresponds to $16 T$ (where $T$ is an approximate 

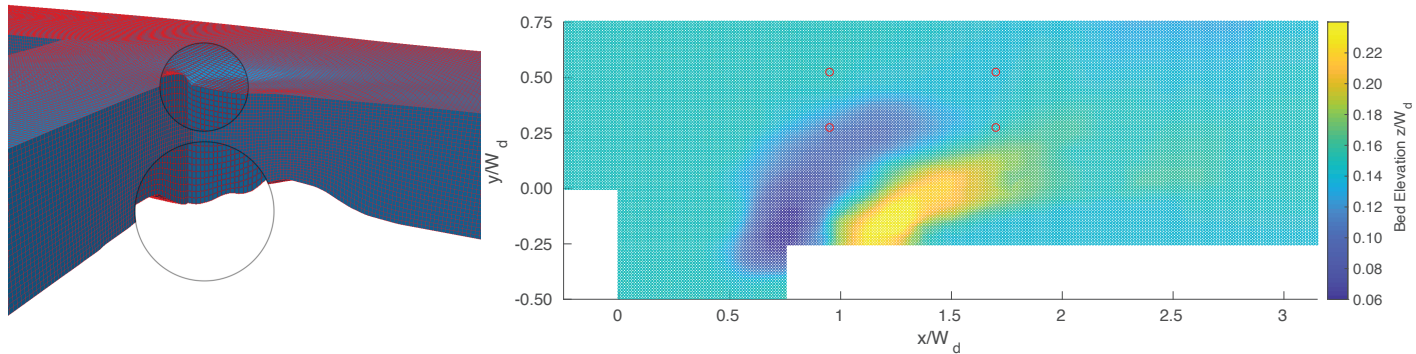

Figure 2. Numerical mesh (left) fitting the curved rigid-lid and the degraded bed (right) obtained by Tang et al. (2018).
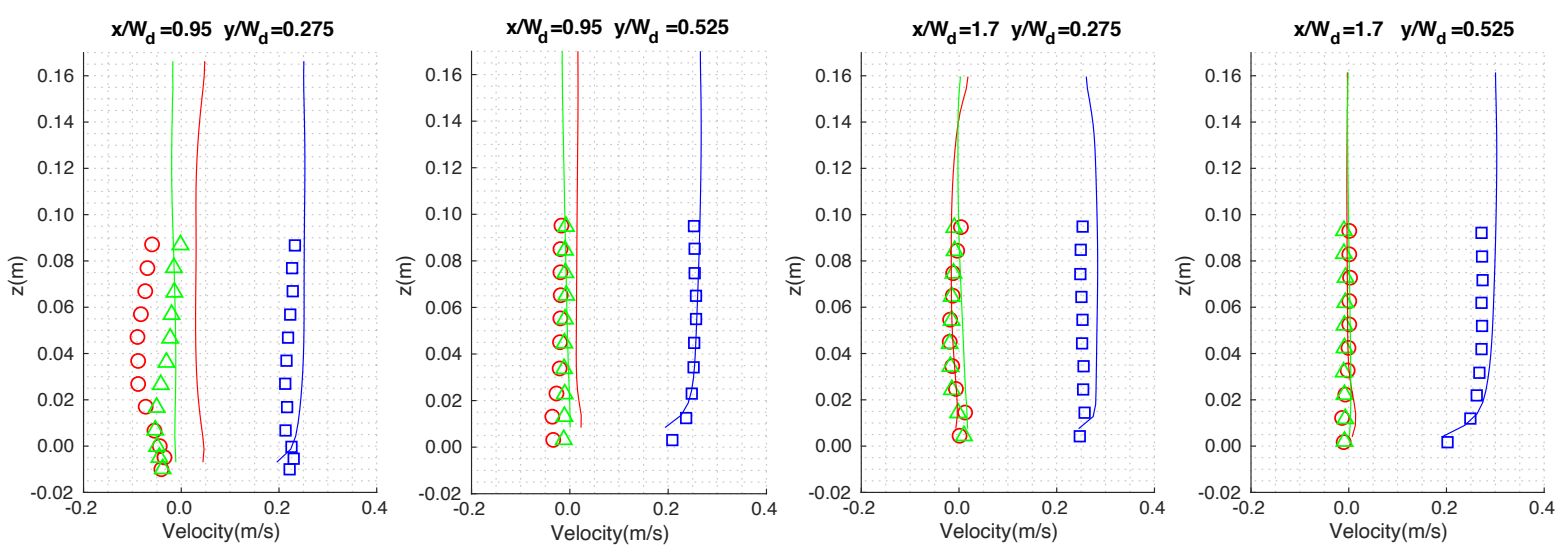

Figure 3. Velocity profiles in the degraded bed case as predicted by the LES simulation and measured by Tang et al. (2018).

flow-through time between the inlet of the tributary and the outlet of the downstream channel). Data collection and time-averaging span an additional 2300s $(\approx 72 T)$ of simulation. The simulations were computed on a $4 \times 12$ cores Intel E5-2680 v.3 (Haswell $-E P @ 2.5 G H z$ ). The total computational cost of a simulation is approximately $5760 \mathrm{CPU}$ hours. Since the numerical domain is decomposed in 48 sub-domains, the real computational time, due to the parallel processing capabilities, is about 120 hours for each simulation.

\section{RESULTS}

\subsection{Validation of the simulations}

For the degraded bed case, Figure 3 shows vertical profiles of the time-averaged velocity components $(u, v, w)$ in a number of discrete points, the location of which is indicated by circles in Figure 2 (right panel). The simulated profiles of the streamwise component $(u)$ show fair agreement with the experimental data. Further improvement near the bed probably requires adopting another approach than wall functions. The cross-wise component $(v)$ and the vertical component $(w)$ are small in absolute value. Therefore, the agreement of simulations and experiments in relative terms and in sign is less well, especially in the most upstream cross-section $\left(x / W_{d}=0.95\right)$. In Tang et al. (2018) no measured passive scalar concentrations are made available for further validation of the simulated passive scalar concentrations against measurements are presented.

\subsection{Time-averaged velocity}

In order to visualize the complex flow in the confluence zone, some streamlines of the simulated timeaveraged velocity fields are indicated in Figure 4, by means of massless tracers injected at an elevation 

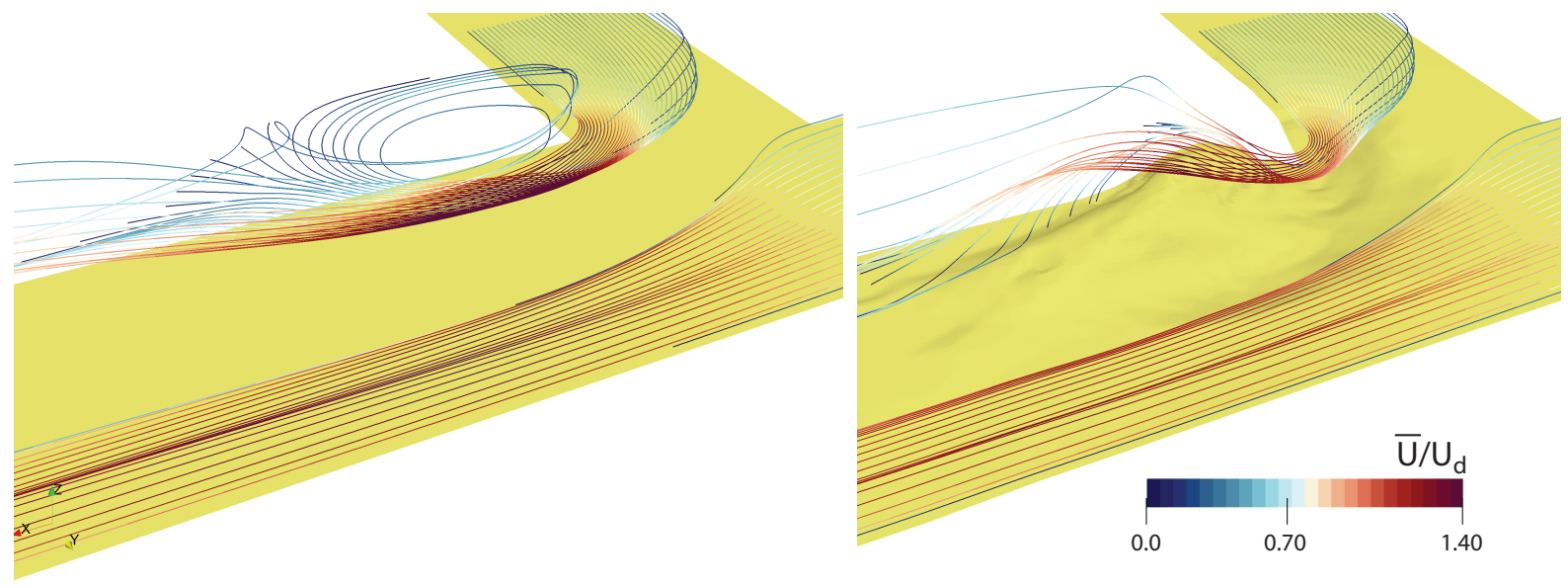

Figure 4. Streamlines (colored by dimensionless time-averaged component $u / U_{d}$ ) of tracers injected in timeaveraged velocity field at an elevation $z / h_{d}=0.05$ at $1 \mathrm{~W}$ upstream of CHZ in main and tributary channel (left: flat bed case; right: degraded bed case).

$z / h_{d}=0.05$ in both the tributary and the upstream main channel. As is clear in both cases, the streamlines originating from the tributary exhibit a flow acceleration towards the downstream confluence corner. In the flat bed case (Figure 4, left), the flow clearly separates at that corner and forms a recirculation zone (RZ). In the degraded bed case, the flow coming from the tributary gets concentrated first in the scour hole and is subsequently guided upstream by the stoss side of the depositional bar, yielding a smaller RZ. The streamlines in Figure 4 being coloured as a function of the streamwise velocity component $(u)$, it is obvious that there is less contraction of the main channel flow in the degraded bed case.

To further describe the time-averaged velocity field, three different cross-sections of the downstream main channel are presented in Figure 5. Each of them is showing both the in-plane component $(v, w)$ by means of vectors and the normal component $(u)$ by colors. The first cross-section, at $x / W_{d}=1$, reveals that the RZ (characterized by $u<0$ ) in the flat bed case is present over the full water column, though the width is reduced near the bed, due to the onset of a secondary flow cell. The bed shear induced by the latter most probably contributes to the scour, which is visible in the corresponding cross-section of the degraded bed case. In the latter case, the RZ has disappeared near the bed. The first cross-section also illustrates the more pronounced lateral velocity vectors and the higher contraction of the main channel flow in case of the flat bed. The second cross-section, at $x / W_{d}=1.5$, shows that the RZ is still present over the full water column in the flat bed case, whereas the RZ has shrunk in the degraded bed case to a small pocket near the bed between the depositional bar and the inner wall of the downstream main channel. This cross-section also indicates that the secondary flow in the degraded bed case significantly differs from the one in the flat bed case, due to the presence of the depositional bar. The third crosssection, at $x / W_{d}=6$, however, indicates that the secondary flow in the two cases is remarkably similar (though there are still differences in the degree of uniformity of the streamwise component). Actually, starting from $x / W_{d} \approx 4$, the secondary flow is found (not shown) to be very similar for the two cases.

\subsection{Turbulent kinetic energy}

For both the flat bed and the degraded bed cases, high values of the turbulent kinetic energy, TKE, are observed in the mixing layer between the merging flows (see cross-section $x / W_{d}=0.2$ in Figure 6 ) and even higher values are found (not shown in this paper) in the shear layer surrounding the corresponding RZ. In the lee of the RZ, the TKE values gradually become weaker and the TKE distribution becomes wider. In the cross-section at $x / W_{d}=6$ of Figure 6 , significantly higher TKE values are found in the flat bed case as compared to the degraded bed case.

\subsection{Mixing of the passive scalar}

Based on the present eddy-resolving LES simulations of the mixing of a passive-scalar-laden tributary flow with a pristine flow coming from the upstream main channel, not only the mean (i.e. time-averaged) 

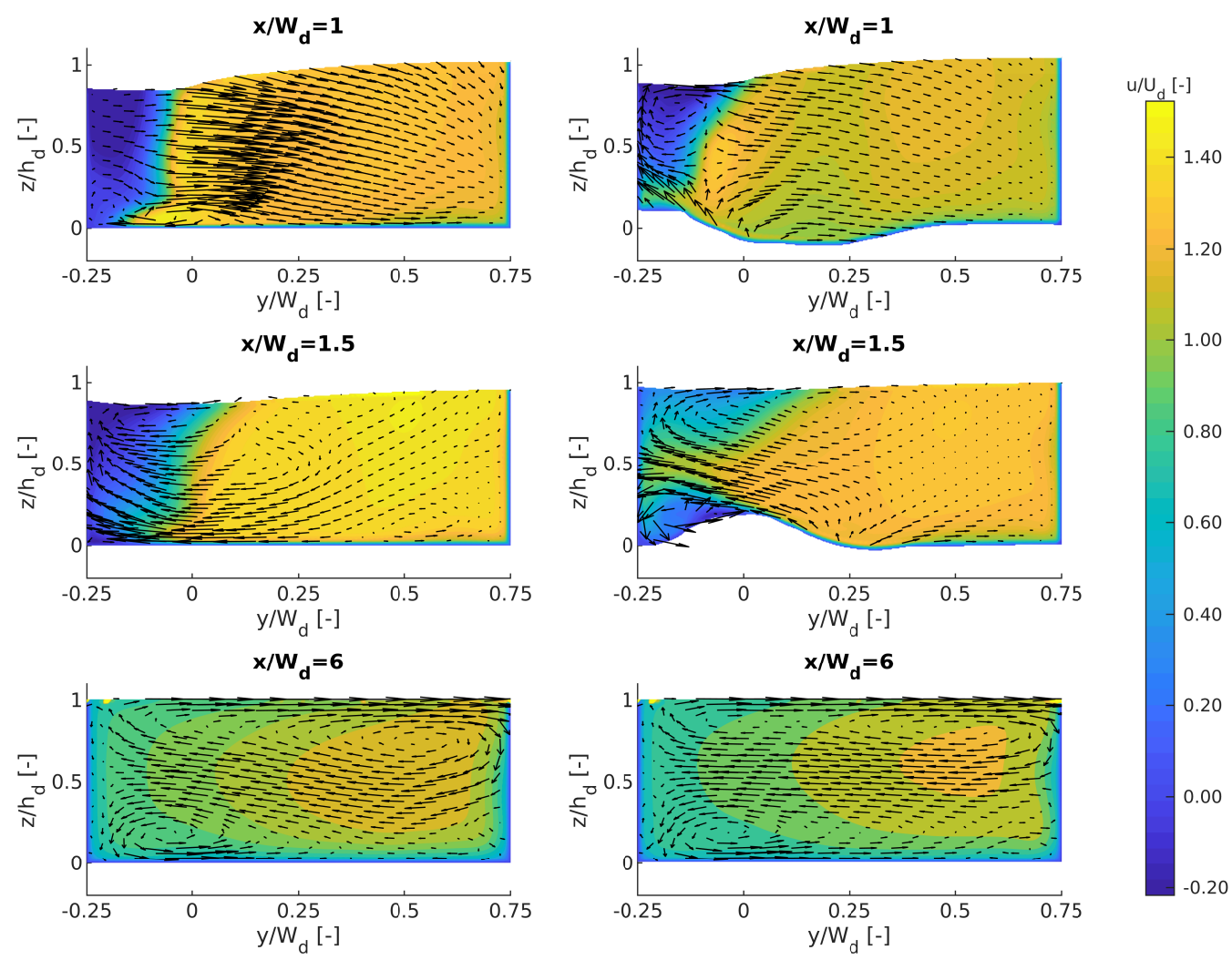

Figure 5. Cross-sections in downstream main channel, showing in-plane component of time-averaged velocity (vectors) and streamwise component of time-averaged velocity (colors) (left: flat bed case; right: degraded bed case).
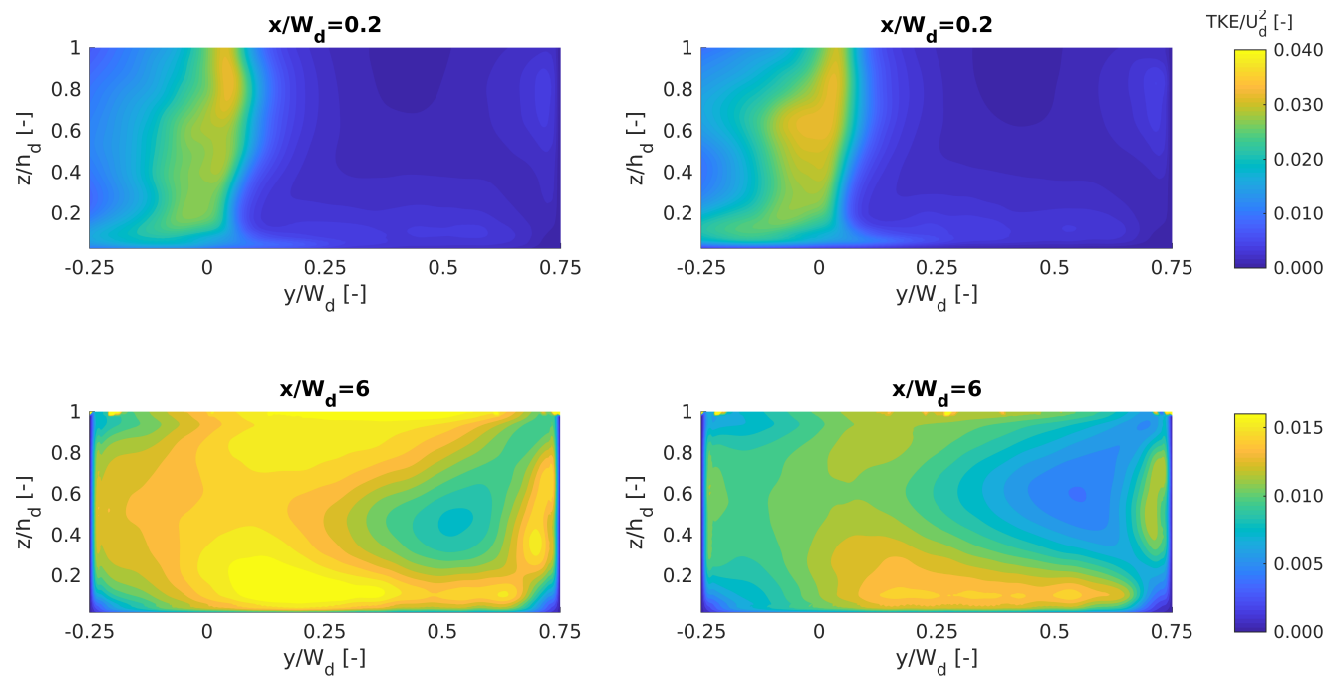

Figure 6. Cross-sections in downstream main channel, showing dimensionless turbulent kinetic energy $T K E / U_{d}^{2}$ (left: flat bed case; right: degraded bed case).

concentration field can be predicted downstream of the confluence, but also the turbulent concentration fluctuations can be quantified. Figure 7 shows the variance $c^{\prime 2}$ in a horizontal plane at mid-depth. The results for the flat and degraded bed cases are quite similar, though the layer with pronounced turbulent concentration fluctuations in between the merging flows is thickening somewhat faster in the degraded 


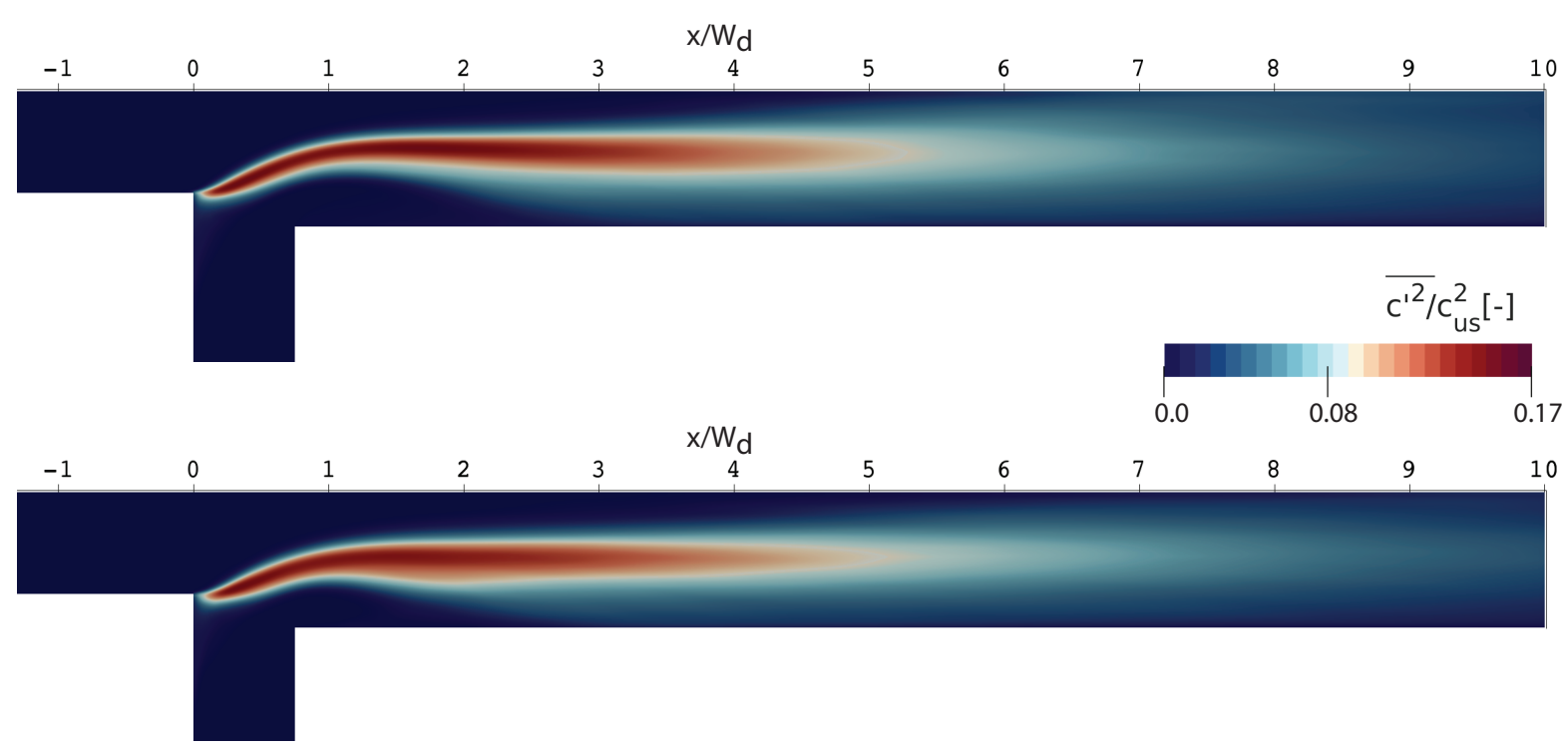

Figure 7. Dimensionless variance of turbulent passive scalar concentration fluctuations $\left(\overline{c^{2}} / c_{u s}^{2}\right)$ in horizontal plane at $z / h_{d}=0.5$ (top: flat bed case; bottom: degraded bed case).

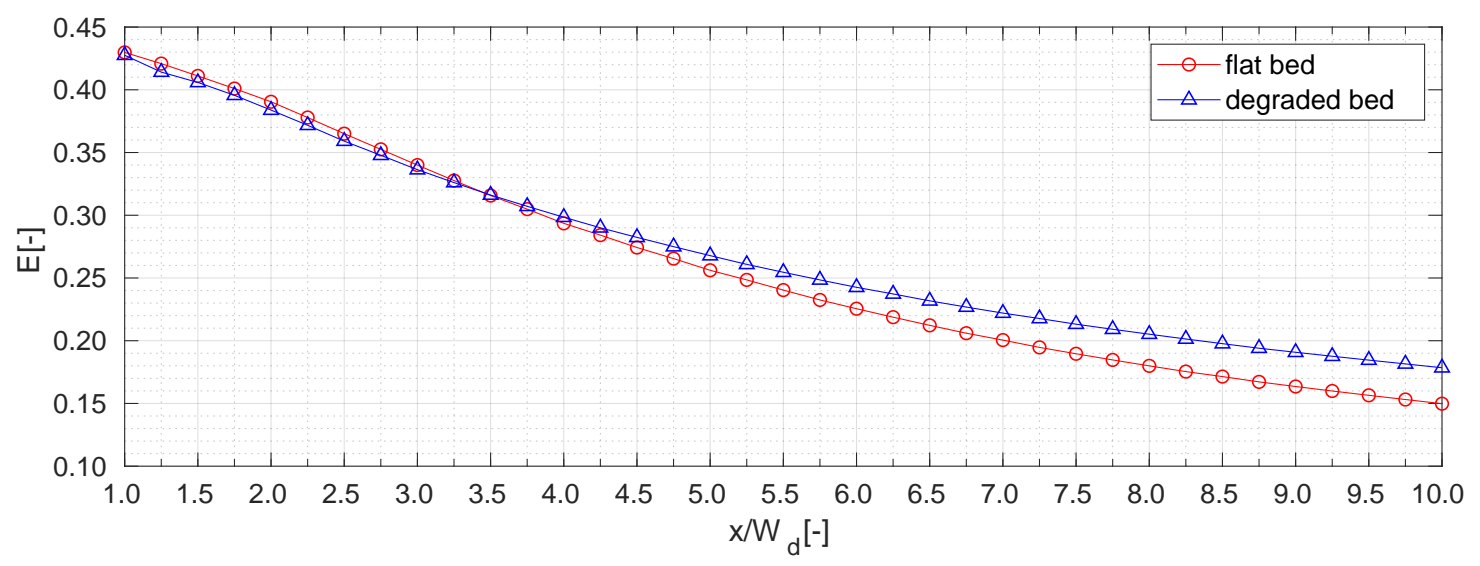

Figure 8. Spatial evolution of non-uniformity of mixing parameter $E$.

bed case and is dissipated slightly faster than in the flat bed case. To quantify the non-uniformity of mixing downstream of the confluence, the dimensionless parameter $E$, used e.g. by Dalmon et al. (2015) and Pouchoulin et al. (2018), is adopted in the present work:

$$
E=\frac{1}{c_{u s}}\left(\sum_{n=1}^{n} \frac{A_{i}}{A}\left(c_{i}-c_{a v g}\right)^{2}\right)^{\frac{1}{2}}
$$

where $\mathrm{c}_{i}$ is the time-averaged concentration in a mesh cell with area $A_{i}, \mathrm{n}$ is the number of mesh cells in a cross-section with total area $\left.A\left(=\sum_{n=1}^{n} A_{i}\right), c_{a v g}\left(=\sum_{n=1}^{n} A_{i} c_{i} / A\right)\right)$ is the cross-sectionally averaged concentration and $c_{u s}$ is the uniform concentration imposed upstream (i.e. at the tributary inlet). In case of complete mixing, the $E$ parameter would assume a zero value. The spatial evolution of $E$ along the downstream main channel is shown in Figure 8. Upstream of $x / W_{d} \approx 5$, the flat bed case shows only a slightly higher non-uniformity of mixing than the degraded bed case. Over the remaining length of the computational domain (which extends up to $x / W_{d}=10$ ), however, the flat bed case seems to experience a somewhat higher degree of mixing. 


\section{DISCUSSION AND CONCLUSIONS}

The recirculation zone (RZ) in the degraded bed case was found to be significantly shorter and less wide or even absent near the bed, due to the complex interaction of the flow with the scour hole and the depositional bar (Figure 4 and Figure 5). The degree of contraction of the main channel flow is also less in the degraded bed case. Despite the aforementioned differences, the non-uniformity of mixing (as derived from the time-averaged concentration field by means of the parameter $E$ ) upstream of $x / W_{d} \approx$ 3.5 (i.e. the zone which contains the major differences in bed morphology between the considered cases) turns out to be only marginally lower for the degraded bed case. This confirms the findings of Tang et al. (2018) with their RANS based simulations (and a different parameter to quantify the non-uniformity of mixing). Note that these findings differ from the enhanced mixing which is usually associated with the presence of bed elevation discordance, as found in studies where a degraded bed in a confluence is schematized to a step in the interface between the (shallower) tributary channel and the main channel (e.g. Bradbrook et al. 2001; Ramos et al., 2019b). Downstream of $x / W_{d} \approx 5$ up till the end of the present numerical domain at $x / W_{d}=10$ (a zone which is not focused on in Tang et al., 2018), the non-uniformity of mixing is found to be somewhat lower for the flat bed case. This finding cannot be attributed to differences in secondary flow patterns, since the latter are found to be very similar for both bed cases as of $x / W_{d} \approx 4$ (see e.g. cross-sections at $x / W_{d}=6$ in Figure 6 ), but differences in TKE levels (see e.g. cross-sections at $x / W_{d}=6$ in Figure 5) and in $\overline{c^{\prime 2}}$ values (Figure 7) may play a role. Finally, it is worth pointing out that the present paper has focused on a single flow condition, characterized by a dominant main channel inflow. Further research is needed to find out whether the present conclusions also hold in case of a tributary dominant inflow condition, like e.g. 'case 2' of Tang et al. (2018) for which the scour hole and the depositional bar have a larger extent.

\section{ACKNOWLEDGEMENTS}

This work was performed using the computational facilities of the HPC infrastructure of Ghent University. The first author is supported by a doctoral research grant of the China Scholarship Council (CSC), with co-funding by the Special Research Fund (BOF) of Ghent University.

\section{REFERENCES}

Best, J.L. 1987. Flow dynamics at river channel confluences: implications for sediment transport and bed morphology. In: Ethridge, F.G., Flores, R.M., Harvey, M.D. (Eds.), Recent developments in fluvial sedimentology. Spec. Publ. Soc. Sediment. Geol. SEPM 39: 27-35.

Biron, P. M., Ramamurthy, A. S., \& Han, S. 2004. Three-dimensional numerical modeling of mixing at river confluences. Journal of Hydraulic Engineering 130(3): 243-253.

Bradbrook, K. F., Lane, S. N., Richards, K. S., Biron, P. M., \& Roy, A. G. 2001. Role of bed discordance at asymmetrical river confluences. Journal of Hydraulic Engineering 127(5): 351-368.

Dalmon, A., Mignot, E., Riviere, N., Lipeme Kouyi, G., Momplot, A., Gonzalez, C., \& Escauriaza, C. 2015. Mélange à l'aval d'une confluence d'écoulements à surface libre. In $22^{e ̀ m e}$ Congrès Français de Mécanique. AFM, Association Française de Mécanique. [in French]

Guillén-Ludeña, S., Franca, M. J., Cardoso, A. H. \& Schleiss, A. J. 2015. Hydro-morphodynamic evolution in a $90^{\circ}$ movable bed discordant confluence with low discharge ratio. Earth Surface Processes and Landforms 40(14): 1927-1938.

Pouchoulin, S., Mignot, E., Rivière, N. \& Le Coz, J. 2018. Numerical simulations on mixing of passive scalars in river confluences. In E3S Web of Conferences River Flow 2018, 40: 5-19. EDP Sciences.

Ramos, P.X., Schindfessel, L., Pêgo, J.P. \& De Mulder, T. 2019a. Flat vs. curved rigid-lid LES computations of an open-channel confluence. Journal of Hydroinformatics 21(2): 318-334.

Ramos, P.X., Schindfessel, L., Pêgo, J.P. \& De Mulder, T. 2019b. Influence of bed elevation discordance on flow patterns and head losses in an open-channel confluence. Journal of Water Science and Engineering.

Rhoads, B. L. \& Johnson, K. K. (2018). Three-dimensional flow structure, morphodynamics, suspended sediment, and thermal mixing at an asymmetrical river confluence of a straight tributary and curving main channel. Geomorphology, 323, 51-69.

Rice, S., Roy, A. \& Rhoads, B. 2008. River confluences, tributaries and the fluvial network. Chichester: John Wiley \& Sons.

Rodi, W., Constantinescu, G. \& Stoesser, T. 2013. Large-eddy Simulation in Hydraulics. Dundee: CRC Press. 
Schindfessel, L., Creëlle, S., \& De Mulder, T. 2015. Flow patterns in an open channel confluence with increasingly dominant tributary inflow. Water 7(9): 4724-4751.

Sleath, J.F.A. (1984). Sea bed mechanics. New York: Wiley.

Talebpour, M. \& Liu, X. 2019 Numerical investigation on the suitability of a fourth-order nonlinear k- $\omega$ model for secondary current of second type in open channels, Journal of Hydraulic Research 57(1):1-12.

Tang, H., Zhang, H., \& Yuan, S. 2018. Hydrodynamics and contaminant transport on a degraded bed at a $90^{\circ}$ channel confluence. Environmental Fluid Mechanics 18(2): 443-463.

Weber, L. J., Schumate, E. D., \& Mawer, N. 2001. Experiments on flow at a $90^{\circ}$ open-channel junction. Journal of Hydraulic Engineering 127(5): 340-350.

Yuan, S., Tang, H., Xiao, Y., Qiu, X., \& Xia, Y. 2018. Water flow and sediment transport at open-channel confluences: an experimental study. Journal of Hydraulic Research 56(3): 333-350. 